\title{
DYNAMIC COST CONTROL METHOD IN PRODUCTION PROCESS AND ITS APPLICATION
}

\author{
Xiaonan Li Tianyou Chai Zhangxiong Yu \\ Research Center of Automation, Northeastern University, Shenyang, China, 110004 \\ Huafu Li \\ Handan Iron \& Steel Group, Heibei, China, 056015
}

\begin{abstract}
This paper, by combining optimal control method with the famous advanced cost management experiences of Handan Steel \& Iron Corporation, presents dynamic cost control method in production process. With the support of information technology, this cost control method emphasises real-time following material fluid and collecting cost data so as to implement on-line cost control. Furthermore, this method has been successfully applied in Handan Steel \& Iron Corporation and has brought about remarkable benefits for the enterprise. Copyright (C) 2002 IFAC
\end{abstract}

Keywords: preset, feed-forward control, decentralized control, predictive control, information technology

\section{INTRODUCTION}

Optimizing comprehensive production index has been one of the means to enhance the ability of the modern enterprise to compete with others. Product cost, especially production process cost of product process cost, is one of the most important comprehensive production indexes.

However, up to now, cost control is usually confined to a kind of 'static control' by cost report forms in the scope of management accounting. In this way, real-time tracing cost information of each working procedure cannot be implemented and thus on-line cost control cannot be realized. Therefore, process cost is higher than the target value and the material consumption in production process is very high.

This paper presents 'a method of dynamic cost control in production process' by combining optimized setting technology with the cost control method of Handan Steel \& Iron Corporation. What should be emphasized is that this dynamic cost control method is under the support of the information technology, such as network, database and so on. The basic idea of this method is to take timely measure to correct adverse cost difference by gathering real-time cost data, carrying though dynamic cost analysis and prediction, employing online cost control. Furthermore, on the basis of this method, we have developed the 'dynamic cost control software'. It is encouraging to find out that its application in actual metallurgy industry could bring about great benefits for the enterprise.

\section{THE DYNAMIC COST CONTROL METHOD}

Optimized Setting method (Chai and Guan, 1998) in complex production process, is to construct presetting model, predictive model, and applies feedforward, feedback and adaptive control method. The famous managing theory — 'simulating market accounting and exercising cost veto', which put forward by Handan Steel \& Iron Corporation, presents a new managing mode for metallurgy industries. The idea of this managing theory is to, by using market price information for reference, draw up reasonable target cost, carry out whole staff -whole process cost management and cost accounting by simulating market price. Associating with the above two methods, the dynamic cost control method presented in this paper means to conduct a seriesconnected, multileveled, feed-forward and feedback cost control system (shown in Fig 1), which 
comprises Product Target Cost Setting Model, Target Cost Decomposing Model, Cost forecasting Model and Cost Controller.

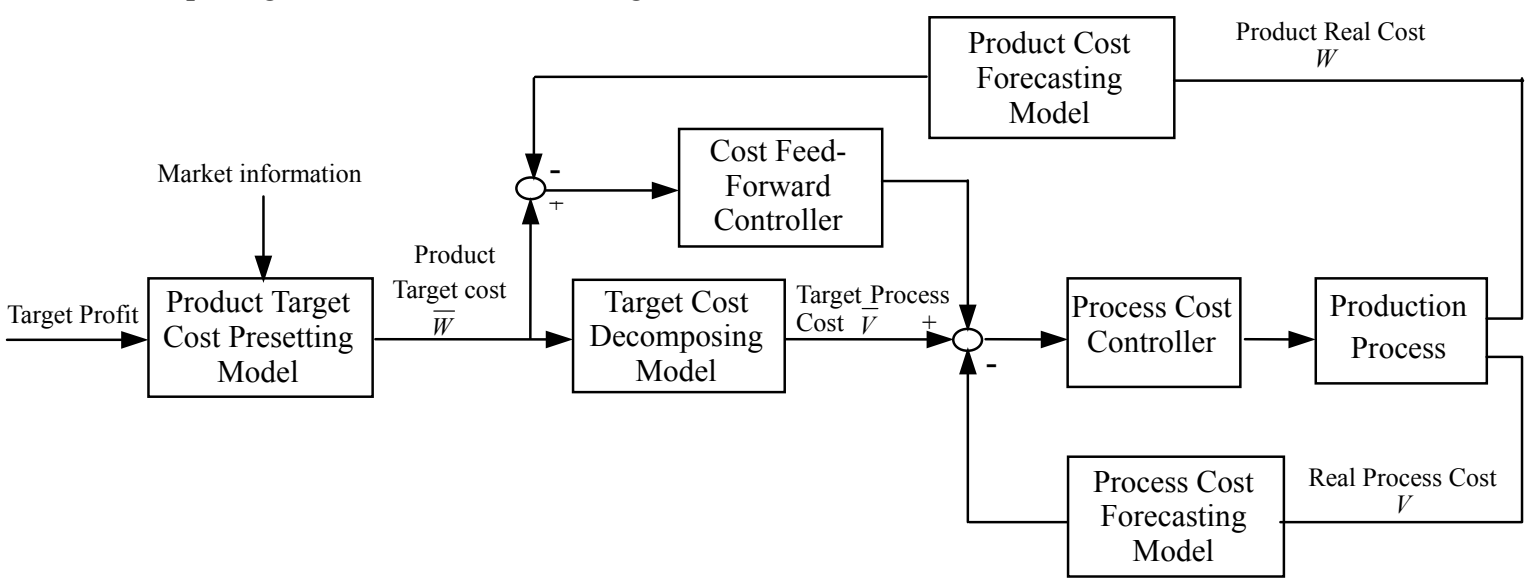

Fig.1 dynamic cost control system
Where:

$$
\begin{gathered}
\bar{W}=\left[\begin{array}{c}
\overline{w_{1}} \\
\overline{w_{2}} \\
\vdots \\
\overline{w_{n}}
\end{array}\right]=\left[\begin{array}{llll}
\overline{w_{11}} & \overline{w_{12}} & \cdots & \overline{w_{1 k}} \\
\overline{w_{21}} & \overline{w_{22}} & \cdots & \overline{w_{2 k}} \\
\vdots & & & \\
\overline{w_{n 1}} & \overline{w_{n 2}} & \cdots & \overline{w_{n k}}
\end{array}\right] W=\left[\begin{array}{c}
w_{1} \\
w_{2} \\
\vdots \\
w_{n}
\end{array}\right]=\left[\begin{array}{cccc}
w_{11} & w_{12} & \cdots & w_{1 k} \\
w_{21} & w_{22} & \cdots & w_{2 k} \\
\vdots & & & \\
w_{n 1} & w_{n 2} & \cdots & w_{n k}
\end{array}\right] \\
\bar{V}=\left[\begin{array}{c}
\overline{v_{1}} \\
\overline{v_{2}} \\
\vdots \\
\overline{v_{m}}
\end{array}\right]=\left[\begin{array}{llll}
\overline{v_{11}} & \overline{v_{12}} & \cdots & \overline{v_{1 l}} \\
\overline{v_{21}} & \overline{v_{22}} & \cdots & \overline{v_{2 l}} \\
\vdots & & \\
\overline{v_{m 1}} & \overline{v_{m 2}} & \cdots & \overline{v_{m l}}
\end{array}\right] V=\left[\begin{array}{c}
v_{1} \\
v_{2} \\
\vdots \\
v_{m}
\end{array}\right]=\left[\begin{array}{llll}
v_{11} & v_{12} & \cdots & v_{1 l} \\
v_{21} & v_{22} & \cdots & v_{2 l} \\
\vdots & & \\
v_{m 1} & v_{m 2} & \cdots & v_{m l}
\end{array}\right] \\
k=\max \left\{\operatorname{dim}\left(\overline{w_{1}}\right), \operatorname{dim}\left(\overline{w_{2}}\right), \ldots, \operatorname{dim}\left(\overline{w_{n}}\right)\right\} \\
l=\max \left\{\operatorname{dim}\left(\overline{v_{1}}\right), \operatorname{dim}\left(\overline{v_{2}}\right), \ldots, \operatorname{dim}\left(\overline{v_{m}}\right)\right\}
\end{gathered}
$$

$\overline{w_{1}}, \overline{w_{2}}, \ldots, \overline{w_{n}}$ are the target cost of Product 1...Product $n, \overline{w_{i 1}}, \overline{w_{i 2}}, \ldots, \overline{w_{i k}}$ are the target cost of items of cost constitution of the Product $i$. If $\operatorname{dim}\left(\overline{w_{i}}\right)=q<k$, then $\overline{w_{q+1}}, \overline{w_{q+2}}, \ldots, \overline{w_{k}}$ are all equal to zero, $w_{1}, w_{2}, \ldots, w_{n}$ are the real cost of all the products, $w_{i 1}, w_{i 2}, \ldots w_{i k}$ are the real cost of items of the cost constitution of the Product $i$.

$\overline{v_{1}}, \overline{v_{2}}, \ldots, \overline{v_{m}}$ are the target cost of each section office or work procedure, $\overline{v_{i 1}}, \overline{v_{i 2}}, \ldots, \overline{v_{i k}}$ are the unit target cost of the cost constitution of one functional division or work procedure. If $\operatorname{dim}\left(\overline{v_{i}}\right)=r<k$, then $\overline{v_{r+1}}, \overline{v_{r+2}}, \ldots, \overline{v_{l}}$ are all equal to zero, $v_{1}, v_{2}, \ldots, v_{m}$ are the real cost of each section office or work procedure, $v_{i 1}, v_{i 2}, \ldots v_{i l}$ are the unit real cost of the cost constitution of one functional division or work procedure.

At first, Product Target Cost Presetting Model sets the target cost of each product by adopting the technique of optimizing setting. The information of market, target profit of enterprise (which has been set), the actual condition of the enterprise, and advanced cost level of similar enterprises are all the reference for Product Cost Setting Model to function. Because of the fact of that, in metallurgy industry, the structure is complex, functions are integrated, and too many factors need to be controlled, we employ decentralizing control strategy in cost control. Thus Target Cost Decomposing Model is conducted to decompose product target cost along the working procedure. In this way it is much easier to implement cost control in each sub-system compared to control the whole system. As a result, the unit target cost (target process cost) is the set target value to each small closed-loop cost control circuit, which is a subsystem of the whole cost control system.

The function of Product Cost Forecasting Model and Process Cost Forecasting Model of each level of work procedure is to gather the data of real cost, process the data by time series model and intelligent algorithms, and provide the interpreted data for cost controller.

The difference of product real cost and product target cost, which are output respectively from Product Cost Forecasting Model and Product Target Cost Setting Model, is input to Cost Feed-forward Controller, and processed by it according to control rules. In addition to the control instruction from Cost Feed-forward Controller, the difference between real process cost from Process Cost Forecasting Model and target process cost of each level of work procedure from Target Cost Decomposing Model is input to Process Cost Controller, and processed by it according to control rules. Then control instruction is sent to production process so as to guide production.

\subsection{Product Target Cost Presetting Model}

Product Target Cost Presetting Model (PTCPM) is to present the reasonable target cost of each product by PLC theory, which is guided by market price. The principle of PLC (Priced-Led-costing) theory is shown in the following form:

Where: $C$ is product target cost, $P_{f}$ is predicted profit,

$$
C=P_{c}-P_{f}-T
$$


$P_{c}$ is product price.

If the target profit (subtracted tax) is fixed, the PLC theory is changed into the following form:

$$
\sum_{i=1}^{m}\left(P_{i}-C_{i}\right) \cdot N_{i} \geq P_{f}
$$

Where: we suppose that there are $m$ kinds of products in the company. $P_{i}$ is the market price of a certain product, $C_{i}$ is product cost, $N_{i}$ is planed yield of the product, $P_{f}$ is the sum of target profit of the company. This formula is the basis for PTCSM to function.

According to the rule of 'setting advanced and reasonable target cost', the product real cost data last year or even old data and all sorts of elements relative to product cost are concerned and analyzed in PTCSM (Christian and Michael, 1997).

\subsection{Target Cost Decomposing Model}

In Target Cost Decomposing Model (TCDM), the product target cost from PTCPM is decomposed into unit target cost according to the product cost construction. Similarly, each unit target cost is decomposed according to the rule, which is shown as the following form:

$$
\frac{\sum_{i=1}^{k} C_{a i} \times N_{i}}{\sum_{i=1}^{k} N_{i}} \leq C_{a}
$$

Where: $C_{a}$ is the target cost of a certain item in the cost construction of a certain level of working procedure. $C_{a i}$ is the target cost of such item in the cost construction of a certain lower level of working procedures. $N_{i}$ is the product yield of a certain working procedures in the lower relative one. $k$ is the number of lower level of working procedure whose cost construction comprises such item. At last, these units of target cost will be the bases for cost analyses and control.

\subsection{Cost Forecasting Model}

Cost Forecasting Model (CFM) compromises Product Cost Forecasting Model and Process Cost Forecasting Model. The function of CFM is to gather the real-time product cost data and real process cost data of each level of working procedures through network, and process these data to interpret useful predictive information for cost controller (Guh, et al., 1999; Ferdinand, et al., 2000b).

In order to implement daily cost control, CFM collects daily real cost data of each working procedures, processes these data by time series model, and provide the useful information to Cost Feed-forward Controller and Cost Controller after cost analyses and forecasting.
At the end of each cycle of the cost monitoring, CFM collects the product cost data and process cost data of the whole period of cost monitoring, and provide such information to Cost Feed-forward Controller and Cost Controller to analyze and execute corresponding reward or punishment.

CFM is one of the keys to realize dynamic cost control. Because of the real-time following and collecting the cost information fluid of products and each working procedure, cost controller can employ on-line cost control to correct adverse cost difference between real cost and the target value. As a result, the usual method of cost control — 'checking out after the matter has happened', is discarded and the product cost can be really reduced.

\subsection{Cost Controller}

The target of cost control is to implement cost control and guarantee that the real cost be limited to a certain scope. In the normal control system, the variable to be controlled is hoped to be the same as the set point. In cost control system, however, the output is hoped to be under the set point, and the bigger the negative difference, the more profit the enterprise will gain if the market price is relatively fixed.

Cost Feed-forward Controller is to, according to product real-time cost information, implement feedforward control concerning about many more factors in the scope of the whole company. The control instructions and decision-making information from Cost Feed-forward will be sent to Cost Controller.

The main function of the cost controller is to implement cost analyses and make decision. During the cycle of cost examination, the cost controller not only analyzes and tries to find out the reasons for the difference between real cost and target value, but also sends out the timely instruction of guidance to correct the adverse difference. As a result, on-line cost control is fulfilled. At the end of cycle of cost examination, the cost controller analyzes real cost data and executes punishment or reward to realize cost afterwards control.

\section{APPLICATION}

Handan Steel \& Iron Group is set up in 1958. It has succeeded in the competition by employing the famous management theory 'simulating market accounting and exercising cost veto', which presents a new management mode for metallurgy industries. The successful implementation of such management mode has brought about immense social benefits as well as great economic benefit for the enterprise. But, because of the reality that most of the work in cost control is completed mainly by handwork. The task is extremely onerous for administrative workers. Furthermore, what is even worse is that real-time following and gathering process cost data, and online cost control cannot be fulfilled in this way. As a result, the effect of cost control is greatly limited. 
Based on the dynamic cost control method presented above, we have developed cost control software for Handan Steel \& Iron Group by adopting information technology of computer network and database. This cost control software has run well and has made great benefits for the enterprise.

\subsection{Software Environment}

\section{Table. 1 Cost control software environment}

\begin{tabular}{|c|c|c|c|}
\hline Hardware & Network & \multicolumn{2}{|l|}{ TCP / IP network } \\
\hline equipment & Server & \multicolumn{2}{|c|}{ COMPAQ-PROLIANT-3000 } \\
\hline & Client computer & \multicolumn{2}{|c|}{$\begin{array}{l}\text { Single media of Legend-PC standard configuration, CPU-PII200 above, } \\
800 * 600 \text { display device, local or network laser/ spay-ink/stylus printer }\end{array}$} \\
\hline \multirow[t]{4}{*}{$\begin{array}{l}\text { Supporting } \\
\text { Software }\end{array}$} & Server & Operating system & $\begin{array}{l}\text { WINDOWNS NT SERVER } 4.0 \text { or later } \\
\text { edition }\end{array}$ \\
\hline & & Internet application & $\begin{array}{l}\text { MS SQL SERVER } 7.0 \text { or later edition, cost } \\
\text { control system software at server }\end{array}$ \\
\hline & Client & Operating system & Windows 95 in Chinese or later edition \\
\hline & & Internet application & $\begin{array}{l}\text { DELPHI5.0 or later edition, cost control } \\
\text { system at client computer }\end{array}$ \\
\hline
\end{tabular}

\subsection{The Functions of Cost Control Software}

The main functions of the cost control software are shown in Fig.2:
Drawing up the planed transmitting price of all sorts of materials, such as raw materials, fuels, water and so on, and products and semi-finished products in the enterprise.

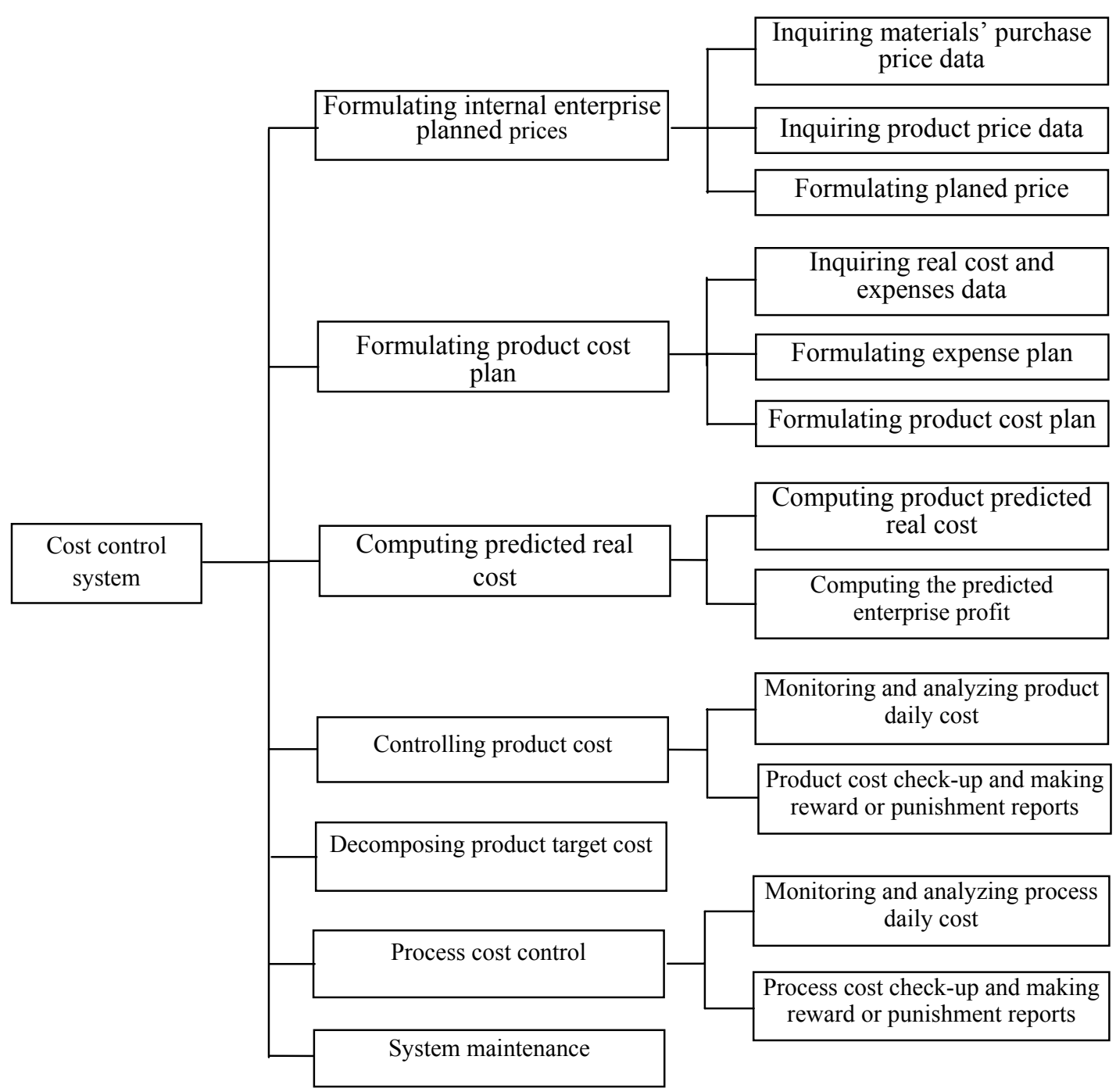

Fig.2 The function tree of cost control software 
Drawing up the target cost of each semi-finished products and products. The user can browse and inquire elaborate cost analyses and data conveniently.
The consumed quantity change curve of continuous casting billet of a certain round steel provided by the software is shown in Fig.3.

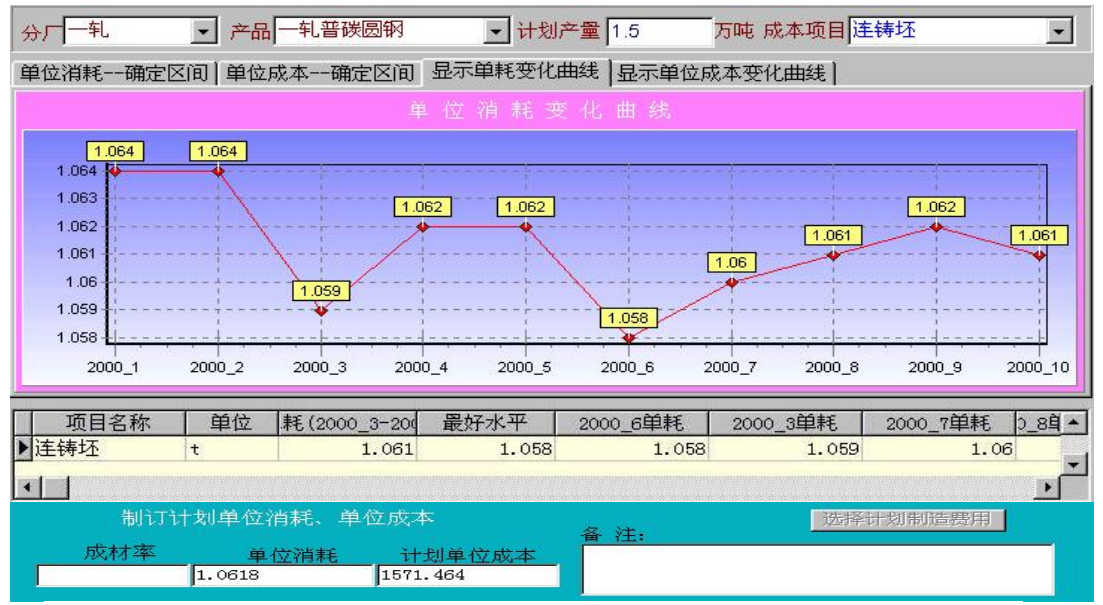

Fig. 3 the screen picture of formulating product cost plan module

Decomposing the product target cost into the unit target cost (process cost) to the functional divisions and workshops.

Daily monitoring real process cost of each working procedure.

Examining and analyzing real cost data, executing punishment and reward in the scope of each working procedure.

Daily monitoring real cost of each product (including semi-finished products) in the scope of the whole enterprise.

Examining and analyzing cost data, executing punishment and reward according to the check-up result in the scope of the whole enterprise.

\subsection{The Network of Cost Control System}

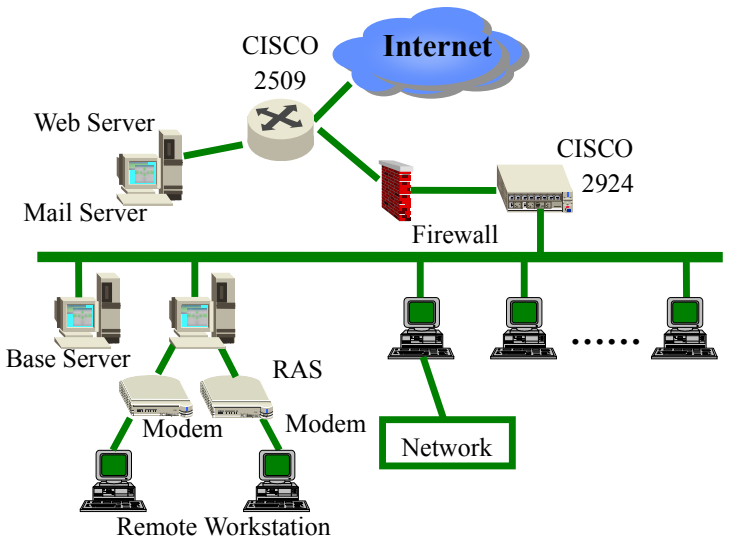

Fig.4 the network of cost control system

\subsection{Application Effects}

The cost control software, which is developed by adopting the information technology based on the method of dynamic cost control presented above, has been applied completely and functioned well in Handan Steel \& Iron Group. There are several characteristics in the software as the following:

To the corresponding cost management departments, the abilities of management and processing has been greatly intensified, and the qualities and efficiency of work have been enhanced. In the past, before the software is applied, the task of working out the product target cost and decomposing target cost will spend a month of time. Furthermore, because of the great mount of data collecting work, the cost plan usually renewed only once or twice a year in the past. But now, after the software has been applied, such task needs only three or four days. The cost plan can be adjusted or renewed at any time according to the actual need.

It is the desirable function of data gathering that guarantees the cost control system to collect the real time and needed data from other systems (Yu, 2000; Cai et al., 1998b). As a result, the efficiency and veracity of the gathering and processing data has been greatly enhanced.

The function of giving advanced and reasonable target cost, by rapid and accurate computing, makes the basis for dig out the potential of reducing cost.

The speeding and accurate information feedback realized the daily or weekly cost monitoring and analyzing, which attributes a lot to the successful implementation of dynamic cost control and reasonable decision-making.

Because the work efficiency has been greatly enhanced, the administrative workers may spend much more time on analyzing cost data and carrying out instigation in the working locale. Therefore, lots of latent severe problems will be uncovered and solved in time to reduce the product real cost.

All these characteristics of the cost control software above will contribute to save cost about RMB 20 
million per year according to economic benefit analyses ( $\mathrm{Li}$ and $\mathrm{Su}, 2000$ ) for the enterprise

\section{CONCLUSION}

The application of the cost control software can formulate the advanced and reasonable product target cost, implement on-line cost control, correct the negative cost difference, and control the real cost below the target value strictly. Therefore, this method of dynamic cost control in production process is advanced and efficient. With the support of information technology, the on-line cost control will be implemented by following the material fluid and cost information fluid, gathering the real time cost data, monitoring and controlling real cost to below the target value. As a result, the product real cost can be really reduced and the profit will be gained accordingly.

\section{REFERENCE}

T.Y.Chai, Shouping Guan (1998), "Object-Oriented Integrated Control Technology of Complex Industrial Processes", 5th IFAC Symposium Low Cost Automation, PS P1, 3-11.

Janssen, Christian, Cain, Michael, "Target selection in process control under asymmetric costs", Journal of Quality Technology $\mathbf{v} 29$ n 4 , Oct1997 ASQC p 464-468 0022-4065

Guh, R.-S, O'Brien, Christopher, "Economical statistical process control using quality cost simulation approach", International Journal of Industrial Engineering: Theory Applications and Practice v 6 n 1, 1999 University of Cincinnati $p$ 48-60 1072-4761

Bloemer, Ferdinand, Guenther, Hans-Otto | Kaminiarz, Bernd, Simulation-aided material flow control and strategic cost analysis of a processing plant, Chemical Engineering and Technology 235, May 2000 Wiley-VCH Verlag GmbH p 401-404 0930-7516

Yu changguo, cost control in ERP, Economist, 2000 (001) (in Chinese)

Zixing Cai, etc., the construction of CIMS based on the integrating of the cost information, Management Information System. 1998.1(in Chinese)

HuaFu Li,Yingmin $\mathrm{Su}$, the report of economic benefit analysis, Handan Iron \& Steel Corporation CIMS863 project acceptance data, 2000.11(in Chinese) 